\title{
COMPARISON OF BONE-IMPLANT CONTACT AND BONE-IMPLANT VOLUME BETWEEN 2D-HISTOLOGICAL SECTIONS AND 3D-SR $\mu$ CT SLICES
}

\author{
Ricardo Bernhardt ${ }^{1 *}$, Eberhard Kuhlisch ${ }^{2}$, Matthias C. Schulz ${ }^{3}$, Uwe Eckelt ${ }^{3}$ and Bernd Stadlinger ${ }^{4}$ \\ ${ }^{1}$ Max-Bergmann-Center for Biomaterials, Technische Universität Dresden, Dresden, Germany \\ ${ }^{2}$ Institute for Medical Informatics and Biometry, Technische Universität Dresden, Germany \\ ${ }^{3}$ Department of Oral \& Maxillofacial Surgery, Technische Universität Dresden, Dresden, Germany \\ ${ }^{4}$ Department of Cranio-Maxillofacial and Oral Surgery, University of Zurich, Zurich, Switzerland
}

\begin{abstract}
Histological imaging is still considered the gold standard for analysing bone formation around metallic implants. Generally, a limited number of histological sections per sample are used for the approximation of mean values of peri-implant bone formation. In this study we compared statistically the results of bone-implant contact (BIC) and bone-implant volume (BIV) obtained by histological sections, with those obtained by X-ray absorption images from synchrotron radiation micro-computed tomography $(\mathrm{SR} \mu \mathrm{CT})$ using osseointegrated screw-shaped implants from a mini-pig study. Comparing the BIC results of 3-4 histological sections per implant sample with the appropriate 3-4 SR $\mu \mathrm{CT}$ slices showed a non-significant difference of $1.9 \%(p=0.703)$. The contact area assessed by the whole $3 \mathrm{D}$ information from the $\mathrm{SR} \mu \mathrm{CT}$ measurement in comparison to the histomorphometric results showed a non-significant difference in BIC of $4.9 \%(p=0.171)$. The amount of the bone-implant volume in the histological sections and the appropriate $\mathrm{SR} \mu \mathrm{CT}$ slices showed a nonsignificant difference by only $1.4 \%(p=0.736)$ and also remains non-significant with $2.6 \%(p=0.323)$ using the volumetric $\mathrm{SR} \mu \mathrm{CT}$ information. We conclude that for a clinical evaluation of implant osseointegration with histological imaging at least 3-4 sections per sample are sufficient to represent the BIC or BIV for a sample. Due to the fact that in this study we have found a significant intra-sample variation in BIC of up to $\pm 35 \%$ the selection of only one or two histological sections per sample may strongly influence the determined BIC.
\end{abstract}

Keywords: Bone formation, metallic implants, imaging, histology.

\author{
*Address for correspondence: \\ Dr Ricardo Bernhardt \\ Max-Bergmann-Center for Biomaterials \\ Technische Universität Dresden \\ Budapester Str. 27 \\ 01062 Dresden, Germany
}

Telephone Number: +49 35146339388

FAX Number: +49 35146339401

E-mail: Ricardo.Bernhardt@tu-dresden.de

\section{Introduction}

The aim of this study was to investigate the use of synchrotron radiation micro-computed tomography $(\mathrm{SR} \mu \mathrm{CT})$ as a means of assessing osseointegration and bone formation around implants. The observation of a direct bone-implant contact after a defined healing period, without interposed fibrous tissue, was first reported by Branemark (1983) and described as osseointegration. The definition of the term osseointegration was clarified by Albrektsson et al. (1981) as the direct contact between a loaded implant surface and bone at a microscopic level.

To analyse undecalcified sections of bone and teeth, Donath and Breuner (1982) developed a method which was also applicable for implants. A sawing and grinding technique enabled the preparation of thin bone/implant sections. These are mostly examined after staining at a thickness of 20-30 $\mu \mathrm{m}$. This method serves as the basis for the assessment of bone-implant contact. Microscopically, a high degree of bony contact along several implant threads provided illustrated evidence of an implant anchored in bone (Albrektsson and Jacobsson, 1987). Although the assessment of the bone-implant contact was also possible with techniques like electron microscopy (Linder et al., 1983; Oyonarte et al., 2005), the histomorphometric evaluation of the bone-implant contact (BIC) was established as the most common method and was applied in the majority of subsequent studies.

After implant placement, primary implant stability is achieved by passive mechanical fixation within the host bone. Subsequent steps of bone resorption and new bone formation enable secondary implant stability through osseointegration. The attainment of osseointegration and secondary implant stability are essential to successful implant treatment (Sennerby et al., 2001). It is the BIC that is essential in creating secondary implant stability. This BIC is usually studied by means of histomorphometry (Johansson et al., 1990). Greater bone contact is generally believed to result in a better implant stability (Park et al., 2005).

However, there is uncertainty whether the analysed histological sections represent the entire osseous situation. Histomorphometry is a destructive method. This limits the analysis to approximately three to four histological sections along the implant length axis, per implant. Microcomputed tomography - a non-destructive technique - uses the absorption of X-rays in order to detect density differences inside solid matter. Due to the fact that this tomography is not a direct imaging technique, the resulting three-dimensional information is generated from a mathematical reconstruction procedure of absorption 

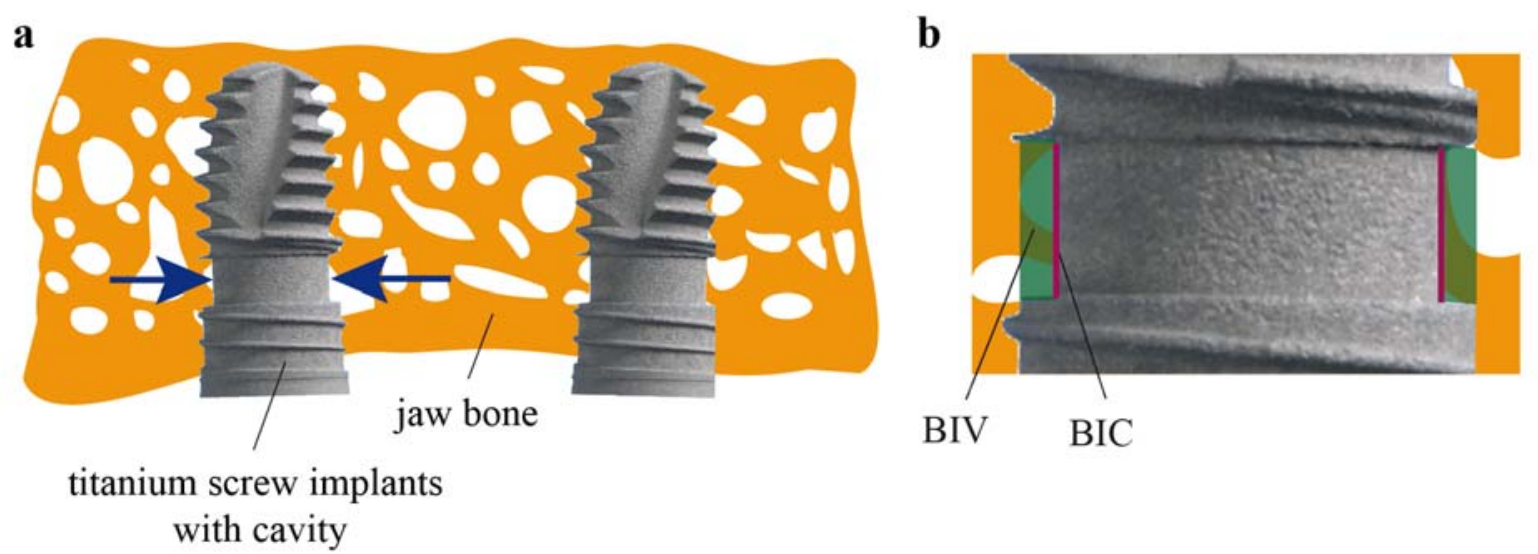

BIV BIC

Fig. 1. (a) Experimental titanium implants with cavities after 8 weeks of healing in the upper jaw bone of minipigs. (b) Regions of interest for the evaluation of newly formed bone (BIC: bone-implant contact, BIV: bone-implant volume).

radiograms from different sample angles. The quality of the representation of the BIC, as well as the bone-implant volume (BIV), mainly depends on the quality of absorption radiograms and is directly associated with $\mathrm{X}$-ray quality.

Limitations of desktop $\mu \mathrm{CT}$ systems for the investigation of the BIC around metal implants are mainly related to metal-induced artifacts. In a recent $\mu \mathrm{CT}$ study the BIC around titanium wires in rat tibia was correlated to findings of backscattered electron microscopy (Liu et al., 2012). In relation to metal-induced artifacts, the authors found for $\mu \mathrm{CT}$ analysis an exclusion zone of $48 \mu \mathrm{m}$ from the implant surface, which prevents any sufficient BIC investigation. In this study we used X-ray photons from the Synchrotron Source BESSY II (Berlin, Germany) for micro-computed tomography. The strength of SR $\mu \mathrm{CT}$ in contrast to desktop $\mu \mathrm{CT}$ systems is the possibility to use monoenergetic X-rays with high intensity in order to eliminate metal-induced artifacts at the implant surface.

Obtaining histomorphometric information from the histological sections, and the three-dimensional information from the tomograms of the same implant/ bone samples enabled us to consider two questions: (i) Are different results obtained for BIC and BIV when comparing 3-4 histological sections (representing one implant/bone sample) to the identical 3-4 virtual slices created by SR $\mu \mathrm{CT}$ volume? (ii) If these results are similar, are differences found in BIC and BIV when comparing the entire data volume from $\mathrm{SR} \mu \mathrm{CT}$ to the 3-4 histological sections?

\section{Materials and Methods}

\section{Study design}

Threaded titanium implants (Fig. 1a) with a diameter of $4.5 \mathrm{~mm}$ and a length of $9.5 \mathrm{~mm}$ were employed. These experimental implants were based on a Xive ${ }^{\circledR}$ (Friadent, Dentsply, Germany) implant. The implant geometry featured a cavity along the implant length axis in order to create a defined area between the implant surface and the round drill hole. This cavity was designed with a defined width of $1.50 \mathrm{~mm}$ and a depth of $0.35 \mathrm{~mm}$. The titanium implants were sandblasted with $250 \mu \mathrm{m}$ corundum, cleaned with isopropanol, rinsed with distilled water, air dried and acid-etched.

From a minipig study evaluating the submerged healing of dental implants in the maxilla (Stadlinger et al., 2011), six implant/host bone samples were randomly selected from different animals after a two-month healing period. The study protocol was approved by the commission for animal studies at the District Government Office, Dresden, Germany. After sacrifice, maxillary en bloc resections were obtained for analysis. The samples were fixed in formaldehyde and dehydrated in a graded series of ethanol. Next, the implants with surrounding bone were embedded in methylmethacrylate (Technovit $9100 \mathrm{neu}^{\circledR}$, Heraeus Kulzer, Wehrheim, Germany), containing the implant nearly centred within the bony tissue.

\section{Synchrotron microcomputed tomography}

In order to perform tomographic measurements, the samples containing the implants were prepared in cylindrical shape with a diameter of about $8 \mathrm{~mm}$. For the non-destructive analysis of newly formed bone within the implant cavity and in close proximity to the implant surface, a SR $\mu \mathrm{CT}$ source at the Berliner Elektronensynchrotron (BESSY II, Berlin, Germany) was used. With monochromatic X-ray energy of $50 \mathrm{keV}, 1500$ radiograms within a $180^{\circ}$ sample rotation were obtained. The reconstruction of the radiograms was performed with a filtered back-projection algorithm for parallel beam. The resolution of the resulting absorption information was $3.58 \mu \mathrm{m}$ in all three dimensions. The six implants so analysed were then used for the histologic and histomorphometric analysis.

\section{Histology}

Undecalcified $100 \mu \mathrm{m}$ thick sections along the length axis of all implants were cut in a parallel direction using a diamond saw microsectioning system (Exakt-Apparatebau, Norderstedt, Germany). For all samples, 3-4 sections were prepared with a cutting distance of about $600 \mu \mathrm{m}$. Only sections representing the implant at full length were included in the analysis. These sections were reduced to 30 $\mu \mathrm{m}$ thickness using grinding techniques on a roll grinder containing sandpaper (Exakt-Apparatebau). Subsequently, 
Table 1. Statistical analysis of the bone-implant contact (BIC [\%]).

\begin{tabular}{cccc} 
sample & $\begin{array}{c}\text { Histo } \\
{[\text { mean } \pm \boldsymbol{S D} \text { ] }}\end{array}$ & $\begin{array}{c}\text { CT-2D } \\
{[\text { mean } \pm \text { SD] }}\end{array}$ & $\begin{array}{c}\text { CT-3D } \\
{[\text { mean } \pm \text { SD] }}\end{array}$ \\
\hline $\mathbf{1}$ & $88.97 \pm 18.37$ & $85.69 \pm 15.08$ & $84.70 \pm 17.72$ \\
$\mathbf{2}$ & $70.56 \pm 20.52$ & $70.60 \pm 18.86$ & $78.66 \pm 20.52$ \\
$\mathbf{3}$ & $62.31 \pm 17.28$ & $62.52 \pm 19.34$ & $65.90 \pm 17.52$ \\
$\mathbf{4}$ & $87.57 \pm 21.67$ & $86.38 \pm 18.40$ & $69.29 \pm 26.23$ \\
$\mathbf{5}$ & $77.42 \pm 35.54$ & $72.37 \pm 33.66$ & $70.01 \pm 25.78$ \\
$\mathbf{6}$ & $84.37 \pm 20.38$ & $82.66 \pm 23.22$ & $73.84 \pm 22.10$ \\
\hline Mean: & $78.42 \pm 23.40$ & $76.29 \pm 22.61$ & $73.74 \pm 22.79$
\end{tabular}

Table 2. Statistical analysis of the bone-implant volume (BIV [\%]).

\begin{tabular}{cccc} 
sample & $\begin{array}{c}\text { Histo } \\
{[\text { mean } \pm \text { SD] }}\end{array}$ & $\begin{array}{c}\text { CT-2D } \\
{[\text { mean } \pm \text { SD] }}\end{array}$ & $\begin{array}{c}\text { CT-3D } \\
\text { [mean } \pm \text { SD] }\end{array}$ \\
\hline $\mathbf{1}$ & $41.63 \pm 13.45$ & $49.52 \pm 15.72$ & $47.17 \pm 16.41$ \\
$\mathbf{2}$ & $39.87 \pm 9.19$ & $43.55 \pm 13.52$ & $46.71 \pm 15.07$ \\
$\mathbf{3}$ & $54.84 \pm 11.08$ & $52.51 \pm 9.69$ & $56.80 \pm 13.72$ \\
$\mathbf{4}$ & $80.41 \pm 11.26$ & $81.20 \pm 11.74$ & $76.21 \pm 9.34$ \\
$\mathbf{5}$ & $60.24 \pm 30.58$ & $59.74 \pm 27.32$ & $61.83 \pm 21.26$ \\
$\mathbf{6}$ & $74.86 \pm 21.30$ & $74.59 \pm 20.71$ & $78.19 \pm 16.16$ \\
\hline Mean: & $59.46 \pm 22.84$ & $60.87 \pm 21.38$ & $61.18 \pm 20.15$
\end{tabular}

Masson-Goldner staining was performed. This staining allowed a clear differentiation of mineralised bone, nonmineralised osteoid and soft tissues. Next, the sections were imaged and analysed using light microscopy (Olympus BX 61, Hamburg, Germany) connected to a computerised system for histomorphometry (Analysis, Soft Imaging Systems, Münster, Germany).

\section{Histomorphometry}

Placing a border at the outer implant surface defined an area within the cavity which served as a region of interest (ROI) for further measurements. The amount of BIC along to the implant length axis within the ROI was measured for every histological section and for two independent lines per section. (Fig. 1b,) Mean values were calculated for each implant. Next, the amount of bone within the ROI was assessed by calculating the percentage of surface area inside the ROI occupied by bone (Fig. 1b, green). Due to the fact that 3-4 histological sections per implant were evaluated, this area is referred to as BIV. Two cavity areas for each slice were independently analysed.

\section{Analysis procedures for 3D volumes from SR $\boldsymbol{\mu C T}$ Threshold of bone values from CT images}

In order to find an appropriate threshold for mineralised bone in the CT volume, the selection was made in best visual agreement with the histological information using identical slices. In the resulting 8-bit grey-level CT images this threshold was set between all absorption values for mineralised bone.

\section{Rotation of SR $\mu C T$-volume to the histological slice} orientation

For the comparison of identical morphological information between histological images and X-ray absorption data, the histologic slice orientation had to be matched in the SR $\mu \mathrm{CT}$ volume. The 3D visualisation software (VG Studio MAX 1.2, Volume Graphics, Heidelberg, Germany) was applied in order to rotate the $\mathrm{SR} \mu \mathrm{CT}$ volume for each sample in the $\mathrm{x}$ - and $\mathrm{y}$-axis to determine the best matching orientation to the corresponding histological section. Next, the entire $\mathrm{SR} \mu \mathrm{CT}$-volume was rearranged according to the detected spatial angles. A z-axis scan through the newly orientated $\mathrm{SR} \mu \mathrm{CT}$-volume was performed for each sample in order to determine the exact position of the other histological sections of the same implant. The BIV for each cavity per $\mathrm{SR} \mu \mathrm{CT}$ slice was calculated in accordance to the histomorphometric assessment. This was measured by dividing the amount of threshold pixels representing bone inside the cavity by the cavity area (Fig. 1b, green area).

Due to the partial volume effect (PVE) from the CT measurement and the pixel interpolation during arbitrary image rotation, a distance of $18 \mu \mathrm{m}$ from the implant surface was defined as the position to obtain values for $\mathrm{BIC}$ in the oriented $\mathrm{SR} \mu \mathrm{CT}$ slices. As there is no straight line or boundary for the assessment of the bone-implant interface in SR $\mu$ CT-slices, the virtual position of the interface was set within the implant adjacent to the rough implant surface. For the BIC measurement this line was shifted 5 pixels $(18 \mu \mathrm{m})$ towards the peri-implant tissues. The histological BIC was measured along a line on the 
outer border of the rough implant surface. The difference between the $\mathrm{SR} \mu \mathrm{CT}$ and the histological BIC line of measurement was about 1-2 pixels (3.5-7 $\mu \mathrm{m})$. Only bone within this fine area of up to two pixels would be excluded in $\mathrm{SR} \mu \mathrm{CT}$ analysis. The percentage of BIC was calculated by dividing the number of threshold bone pixels along the surface of measurement by the total length of this line.

For further comparison between histological sections and $\mathrm{SR} \mu \mathrm{CT}$ slices the following abbreviations for CT data will be used. $C T-2 D$ : for the selected 3-4 slices in the $\mathrm{SR} \mu \mathrm{CT}$ volume that are identical to the 3-4 histological sections. $C T-3 D$ : for all slices in the $\mathrm{SR} \mu \mathrm{CT}$ volume orientated in the histological cutting direction of the 3-4 histological sections.

Extraction of the implant contact surface from SR $\mu C T$ data The three-dimensional character of the SR $\mu \mathrm{CT}$ data enables visualisation of the bone-implant contact as a surface (BIC for CT-3D). Following a manual positioning of the ROI for a selected slice, a fixed position of the bone/ implant interface along the $\mathrm{z}$-axis in $\mathrm{SR} \mu \mathrm{CT}$ slices allows an identical ROI position for the missing slices. In the original $\mathrm{SR} \mu \mathrm{CT}$-volume, the longitudinal implant axis was not equal to the z-axis of the volume. With a digital rotation algorithm (Image Programming Language, SCANCO Medical, Brüttisellen, Switzerland) both axes were superimposed (Fig. 2a). With the help of ImageJ (National Institutes of Health, Bethesda, MD, USA) the concentric peri-implant image values (Fig. 2b) were projected to a secondary data volume (Fig. 2c). Within this rearranged data volume, the z-axis is related to the distance from the implant. A scan along the $\mathrm{x}$ - or $\mathrm{y}$-axis of the rectangle gives information about the amount of bone around the implant and along the implant z-axis respectively. At a constant distance of $18 \mu \mathrm{m}$ to the cylindrical implant surface, found as described in the SR $\mu \mathrm{CT}$ image, a circle with a width of three pixels $(11 \mu \mathrm{m})$ served to define the region of interest (ROI). After the ROI projection (Fig. 2c), a median value was calculated for the superimposed three pixel layers. For the comparison with histomorphometry, a line scan along the projected rectangle (Fig. 2e, ROI 2) served to evaluate the BIC (CT-3D) of the samples.

\section{Statistical analysis}

As a first step, corresponding BIC/BIV values were used to show the regression line between histological and $\mathrm{SR} \mu \mathrm{CT}$ results. Further, a $95 \%$ confidence band for the regression line was applied. The plot was completed by a line of equality. Regression parameters were calculated to describe the linear relation of histological measurements and SR $\mu \mathrm{CT}$-measurements. In a second step, the differences between the histological values and the corresponding SR $\mu \mathrm{CT}$-values were plotted against their mean (Bland-Altman plot). This plot was completed by the limits of agreement and the regression line together with the $95 \%$ confidence band for the regression line. In a third step, all measured values were analysed by a linear mixed model. This allows the modelling of a fixed effect for the measurement methods and of two variance components associated with the variance of implants (intersample variability) and the residual variance (intra-sample variability). All analyses were accomplished with SPSS 17.0 for Windows (SPSS, Inc., Chicago IL, USA).

\section{Results}

\section{Clinical results}

The animals showed normal wound healing at the time of sacrifice. No implant was lost within the selected animals. All six implants healed submerged without exposure.

\section{Histological results}

3-4 histological middle sections (Fig. 3a) that represented the implant at full length could be obtained per implant. The Goldner staining clearly differentiated mineralised bone structures, non-mineralised osteoid, and bone marrow. The peri-implant bone structure consisted of lamellar bone architecture. The implant cavities were filled with newly formed bone. All implants were osseointegrated. The histomorphometric analysis of BIC and BIV is shown in Table 1 and Table 2.

\section{SR $\mu \mathrm{CT}$ - Visualisation and results of the selections}

The measurement position of the synchrotron beam was nearly perpendicular to the implant length axis. The visualisation of the threshold grey values of the raw data from SR $\mu \mathrm{CT}$ for implant and bone (Fig. 3b) showed a clear representation of the spatial bone morphology close to the implant surface within the cavity. No effects from $\mathrm{X}$-ray scattering or ring artifacts were found in the images. The PVE between the titanium implant surface and the peri-implant tissue was measured with a line scan from implant to bone values. A distance of 5 pixels $(18 \mu \mathrm{m})$ from the maximum titanium absorption values was found to be optimal for the CT-2D analysis of the BIC and BIV. Fig. 4 illustrates that it was possible to locate the $\mathrm{SR} \mu \mathrm{CT}$ slices to the corresponding histological sections.

\section{Correlation of BIC histomorphometry and CT-2D, CT-3D}

For each histological section, two independent areas of measurement, one on each side of the implant were evaluated (Fig. 1b). Standard statistical parameters of BIC for histomorphometry, CT-2D and CT-3D for the samples are shown in Table 1. With the described statistical models two different cases were evaluated. First, a paired analysis of the BIC between 3-4 histological sections with the appropriate CT-2D slices (BIC-2D) and secondly a comparison between the histological sections and the CT3D information (BIC-3D). Figs. 2d-e show the result of the digital projection algorithm for BIC-3D in the implant cavity.

In the BIC-2D a non-significant bias of $+1.9 \%(p=$ 0.703 ) between histomorphometry and $\mathrm{SR} \mu \mathrm{CT}$ data was found. The inter-sample variability was $8.2 \%(p=0.298)$, and an intra-sample variability of $21.7 \%(p<0.0005)$ was calculated between both methods. Using the entire range of information from $\mathrm{SR} \mu \mathrm{CT}$ between both methods, a non-significant bias in BIC-3D of $+4.9 \%(p=0.171)$ was found. Here an inter-implant variability of $6.8 \%(p$ $=0.124)$ and an intra-implant variability to $21.9 \%(p<$ $0.0005)$ was calculated. 
$\mathbf{a}$

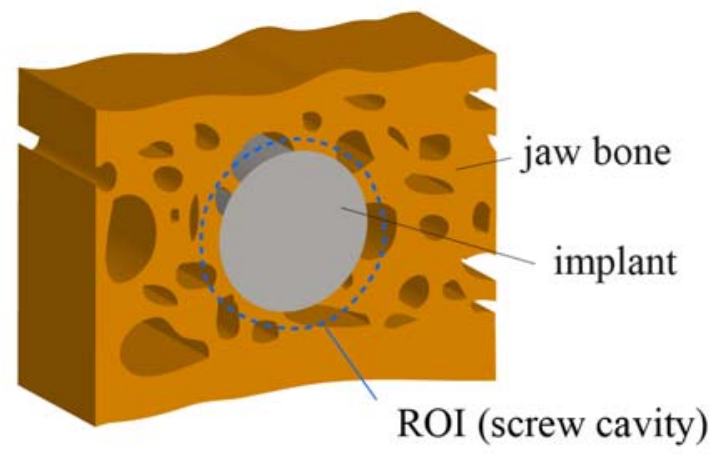

$3 D$ CT-image b
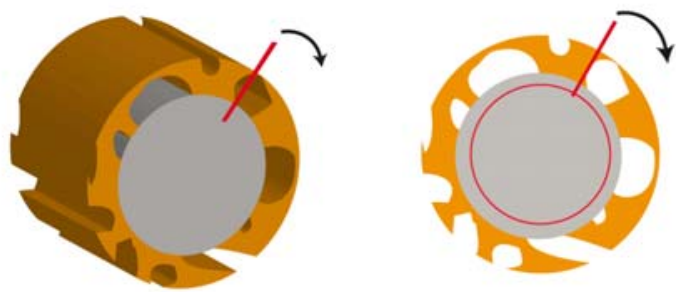

implant cavity projection

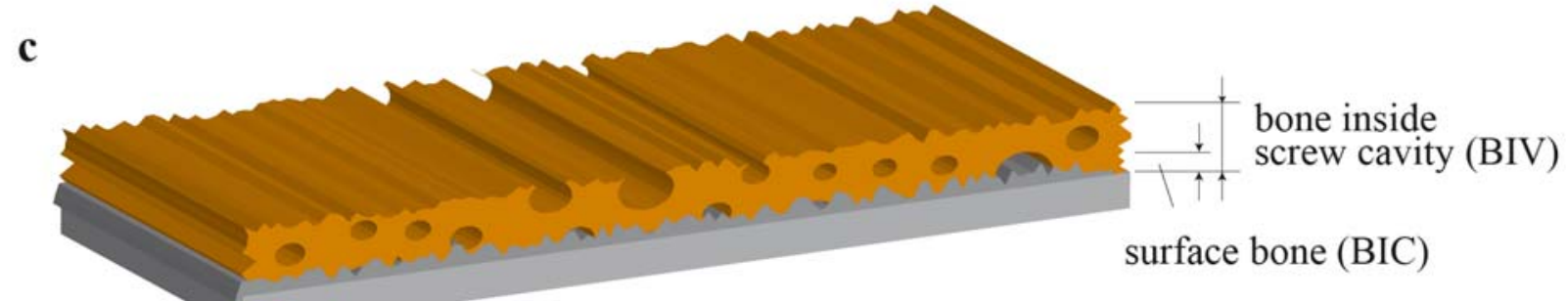

projection area
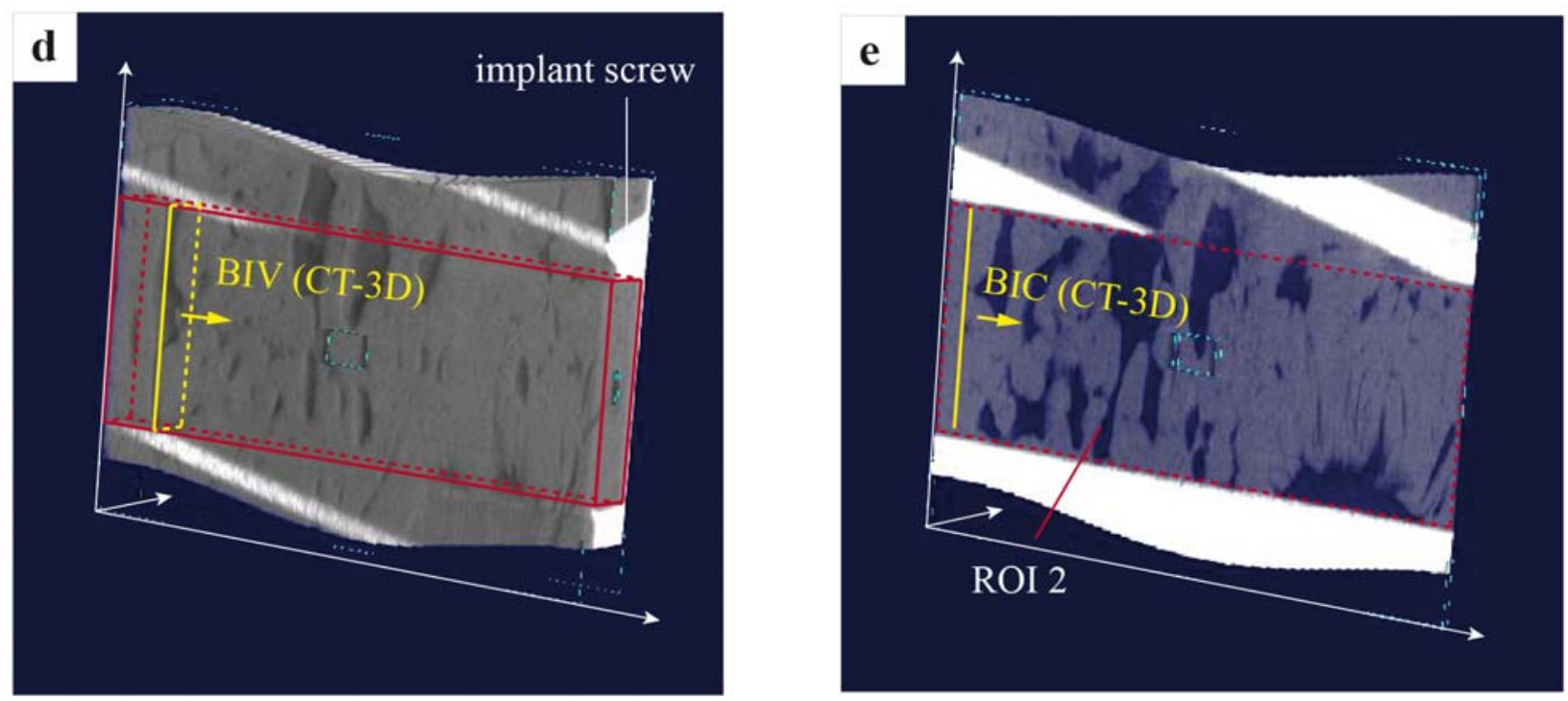

Fig. 2. The rearrangement of SR $\mu \mathrm{CT}$ absorption data using the cylindrical implant geometry is illustrated. (a) First, an alignment between implant longitudinal axis and the $\mathrm{z}$-axis of the $\mathrm{SR} \mu \mathrm{CT}$ volume was performed. Then, a circular ROI around the implant was manually set for a selected slice. (b) The projection algorithm collects the image values along the ROI and reorganise these values to straight lines. (c) With the procedure on all aligned SR $\mu \mathrm{CT}$ slices results in an even morphology of peri-implant bone. (d) A 3D representation of the projected bone implant volume inside the titanium screw cavity shows the result of the procedures. (e) An $11 \mu \mathrm{m}$ thick slice of mineralised bone values at $18 \mu \mathrm{m}$ from the defined implant surface for SR $\mu \mathrm{CT}$ (ROI 2) is shown. This gives real information on the entire bone-implant contact (BIC-3D). 

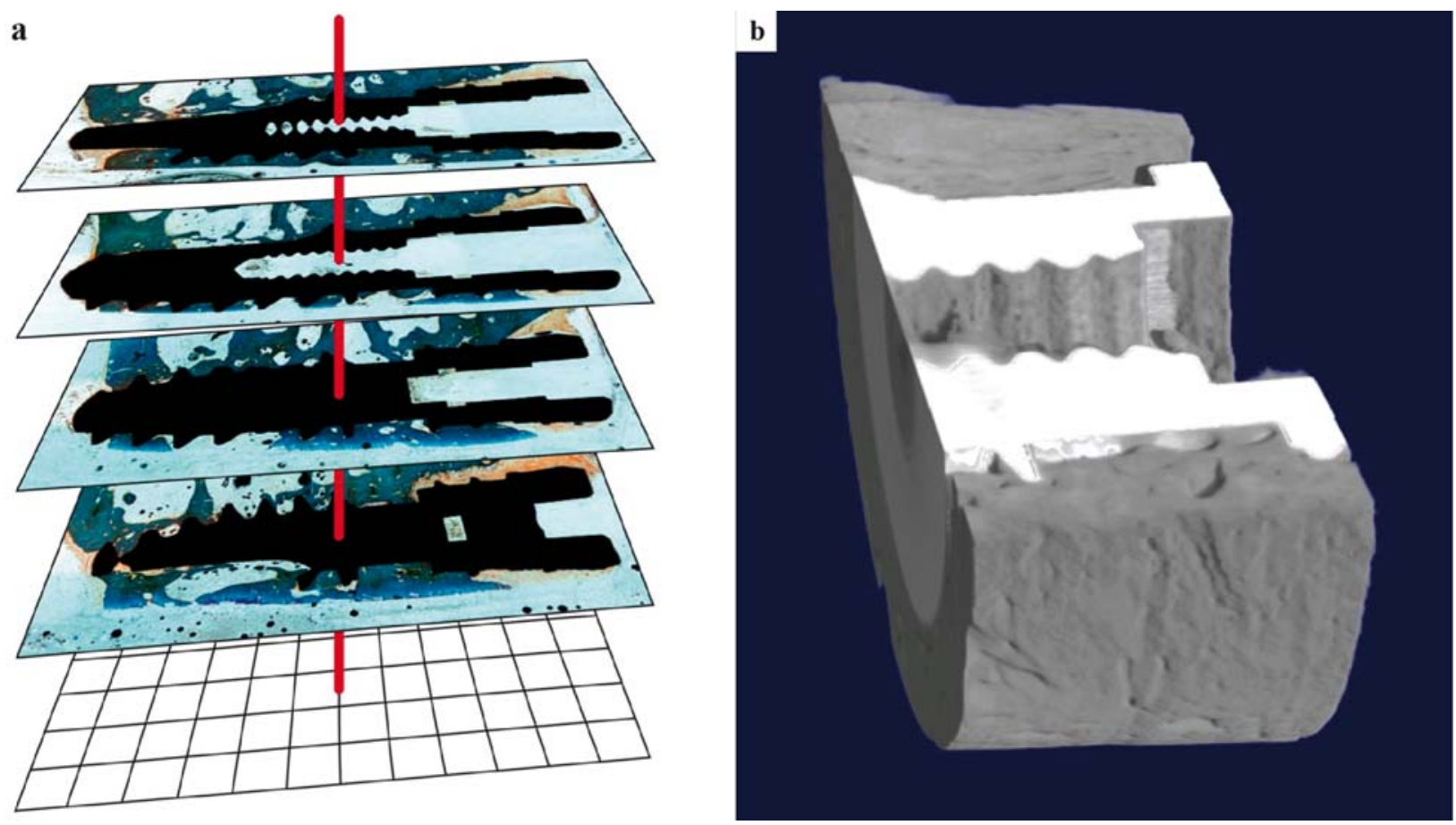

Fig. 3. Generally, morphological information of bone tissue can be assessed by light microscopy of stained histological sections (a), and non-destructively using filtered reconstructed absorption data from SR $\mu$ CT measurements (b). The three-dimensional absorption values of fully mineralised bone are coloured grey and the values for titanium are coloured white.

The linear regression coefficient $(\mathrm{R})$ of the BIC between CT-2D and histomorphometry was calculated with $\mathrm{R}=0.968$ (Fig. 5a). With the Bland-Altman-Plot from Fig. 5b, there is no evidence of a proportional error related to the amount of detected BIC between $\mathrm{SR} \mu \mathrm{CT}$ and histomorphometry. The regression coefficient $\mathrm{R}=0.50$ ( $p$ $=0.883$ ) is not significantly different from zero. Reducing the statistical model to a constant bias, thus revealing systematic errors, results in a significantly higher BIC for histomorphometry compared to $\mathrm{SR} \mu \mathrm{CT}$ with $2.4 \%$ ( $p=$ 0.014).

\section{Correlation of BIV histomorphometry and CT-2D, CT-3D}

Figs. 6a-d illustrates the change of BIV in selected samples comparing histological and CT-3D data in the same diagram. An overall agreement between histomorphometry and CT-2D data for BIV is seen. The standard statistical parameters of BIV for histomorphometry, CT-2D and CT-3D for the samples are shown in Table 2. Statistical models were identical to the BIC calculation. A paired model (BIV-2D) and a model using all information from $\mathrm{SR} \mu \mathrm{CT}$ (BIV-3D) were distinguished. For all samples two independent areas of measurement per section/slice were used.

For BIV-2D a non-significant bias of $-1.4 \%(p=0.736)$ between histomorphometry and CT-2D was found. The inter-sample variability was $14.7 \%(p=0.080)$ and the intra-sample variability was $17.6 \%(p<0.0005)$. For the BIV-3D the bias between histomorphometry and SR $\mu$ CT was $-2.6 \%(p=0.323)$, but remained non-significant. The inter-sample variability in the BIV-3D was $13.7 \%(p<$ $0.0005)$ and the intra-sample variability was $15.8 \%(p<$ $0.0005)$.

A linear regression coefficient $(\mathrm{R})$ of BIV between $\mathrm{CT}$ $2 \mathrm{D}$ and histomorphometry with $\mathrm{R}=0.831$ was calculated (Fig. 5c). Using the Bland-Altman-Plot of Fig. 5d, there is no evidence of a proportional error related to the amount of detected BIV between SR $\mu \mathrm{CT}$ and histomorphometry. The regression coefficient $\mathrm{R}=0.07(p=0.491)$ is not significantly different from zero. Reducing the statistical model to a constant bias shows a non-significant $1.4 \%$ ( $p$ $=0.519$ ) lower BIV for histomorphometry compared to $\mathrm{SR} \mu \mathrm{CT}$.

\section{Discussion}

The aim of this paper was to compare the histomorphometric features of osseointegrated titanium implants with threedimensional information from high-resolution SR $\mu \mathrm{CT}$ measurements. Due to the fact that $\mathrm{SR} \mu \mathrm{CT}$ tomograms are obtained from the absorption of X-ray photons, we analysed whether this spatial data, representing the peri-implant tissue, was comparable to microscopic two-dimensional histological information. To assess this question, BIC and $\mathrm{BIV}$ values from histological sections were compared to $2 \mathrm{D}$ - and $3 \mathrm{D}-\mathrm{SR} \mu \mathrm{CT}$ slices. The histomorphometric measurement of the BIC of osseointegrated implants is the standard procedure for the evaluation of bone formation on an implant surface. High BIC values are considered to be a prerequisite for implant stability, which clinically 

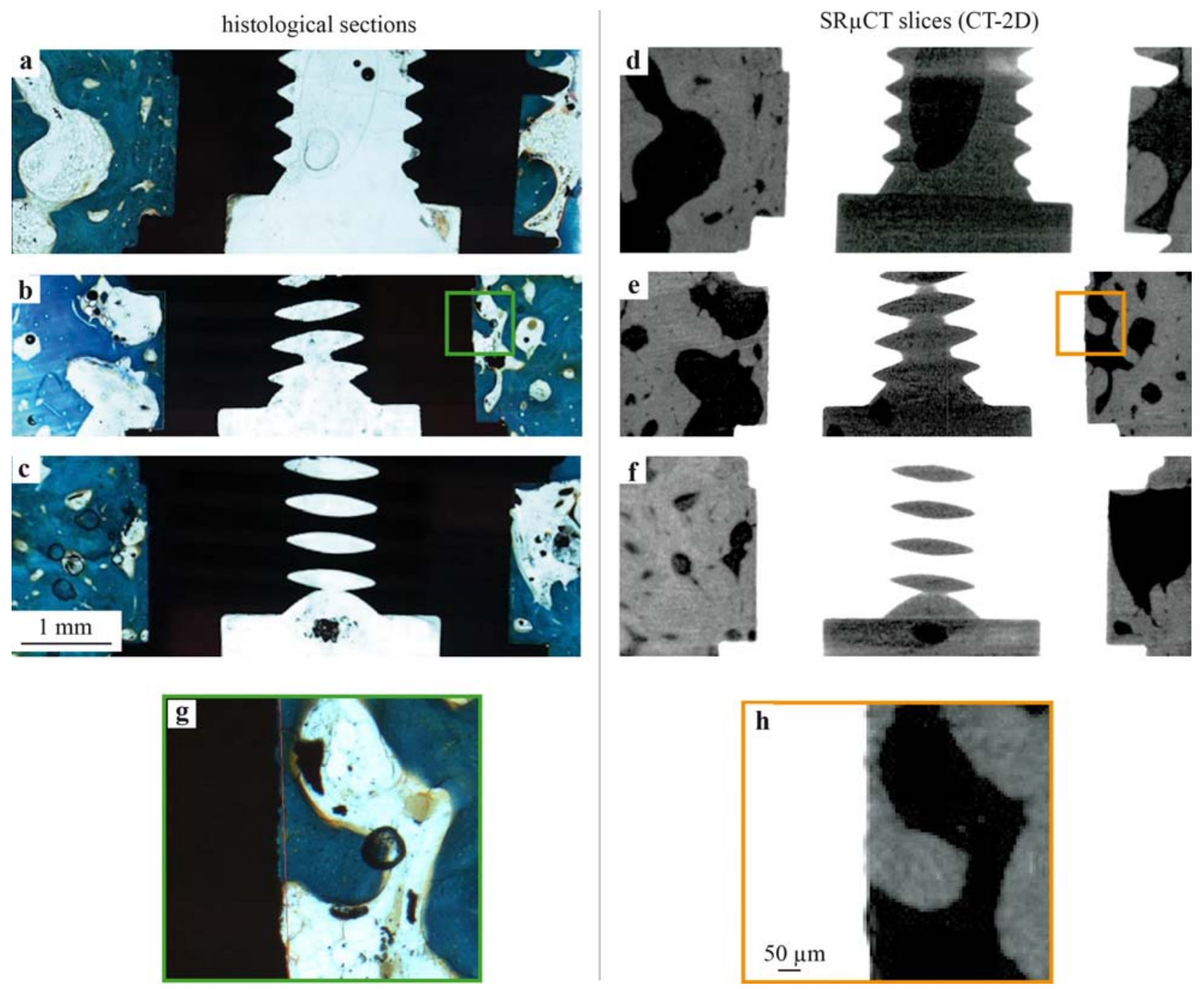

Fig. 4 A prerequisite for a paired statistical analysis between histomorphometry and SR $\mu \mathrm{CT}$ results is the location of the histological section within the SR $\mu$ CT-volume. (a-c) Selected histological sections displaying Masson-Goldnerstained bone tissue in the cavity. (d-f) The appropriate SR $\mu$ CT slices (CT-2D) with absorption values filtered for mineralised bone and titanium show a good morphological agreement for the bone close to the implant surface. (g-h) The image magnifications show a clear representation of the implant interface without metal-induced artifacts for the SR $\mu \mathrm{CT}$ measurement.

enables functional dental reconstruction. Studies evaluating new implant surfaces assess this parameter. In particular, differences in BIC between test and reference surfaces are statistically analysed to compare their osteogenic potential.

In this study, we performed histomorphometric and tomographic analysis of the BIC and BIV of six implant/ bone samples. For the comparison of up to four histological sections (two areas of measurement per histological section) representing a single implant to the CT-2D data, we found a small, non-significant difference in BIC of 1.9 $\%(p=0.703)$. This situation changed when comparing the CT-3D information to the histological sections. The difference in the BIC rose to $4.9 \%$ and was found to be non-significant $(p=0.171)$. The bias of BIC between histomorphometry and CT-3D data was rather small, taking into consideration that the bone contact may vary a lot between all implant surfaces. Comparing the inter- and intra-sample variability of the BIC of both methods, the inter-implant difference was non-significant and decreased by only $1.4 \%$ from BIC-2D to BIC-3D. Furthermore we found a maximum intra-sample BIC standard deviation of $\pm 35.5 \%$ for histomorphometric measurements. This was comparable to the CT-2D situation $( \pm 33.7 \%)$. Considering that many studies use two (or even only one) histological sections per implant, the results of this study illustrate the fact that the selection of the histological section may strongly influence the determined BIC.

In contrast, the intra-sample variability of the BIC between histomorphometry and SR $\mu \mathrm{CT}$ differed significantly with $21.7 \%$ and increased only slightly from BIC-2D to BIC-3D to $21.9 \%$. With the statistical variance model (Fig. 5b) we found in a small but significant systematic error of $2.4 \%(p=0.014)$, which leads to higher BIC values for histomorphometric measurement. While $\mathrm{SR} \mu \mathrm{CT}$ needs a strong contrast for the detection of bone tissue directly at the implant surface, only fully mineralised 
a

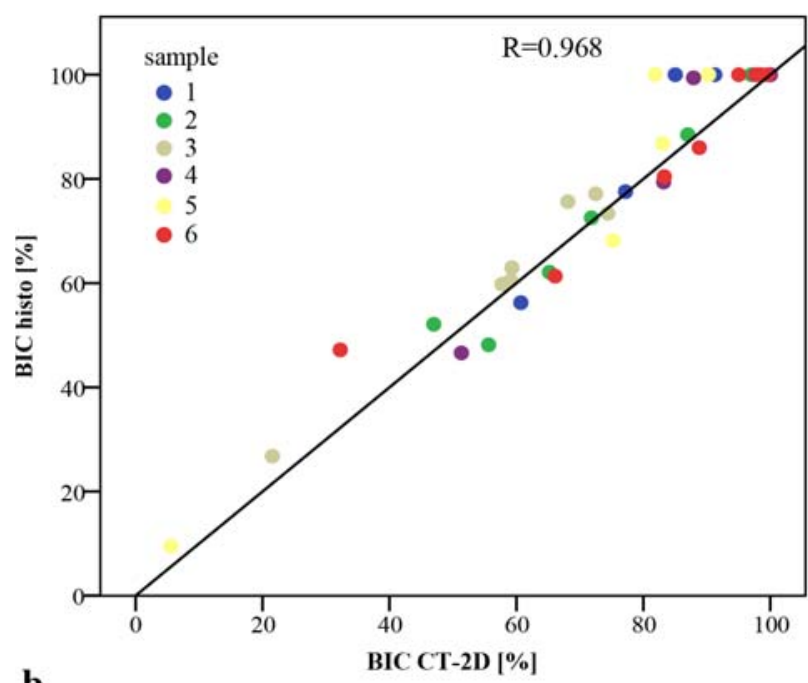

b

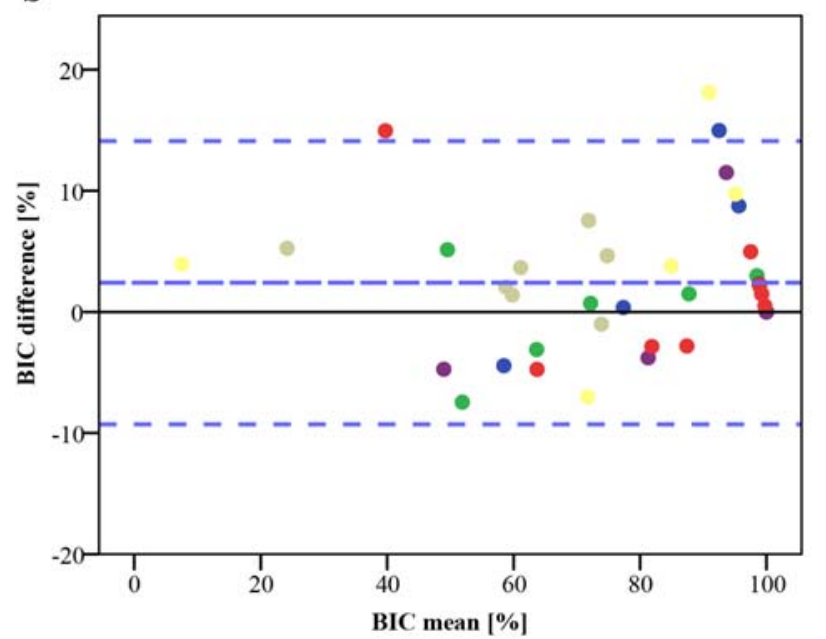

c

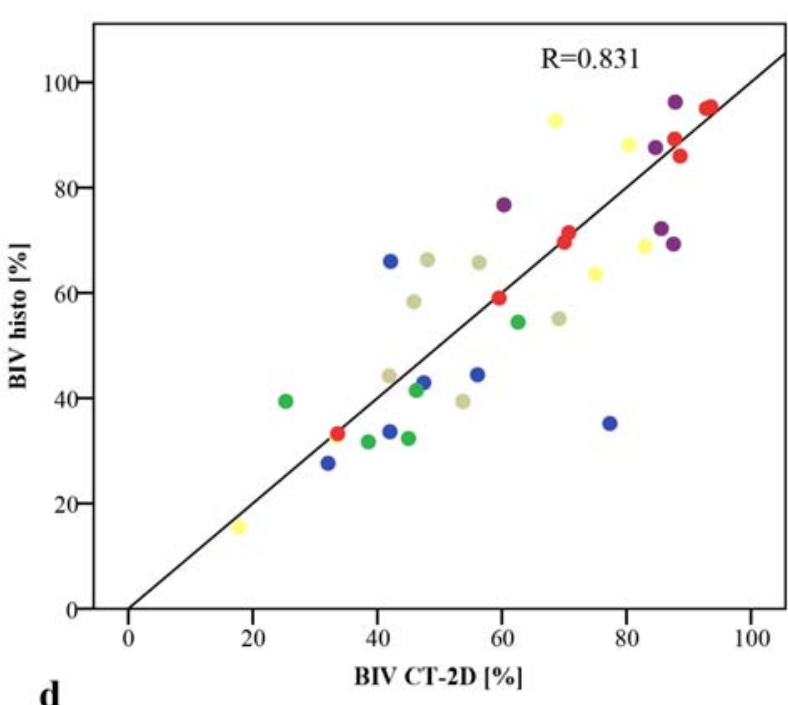

d

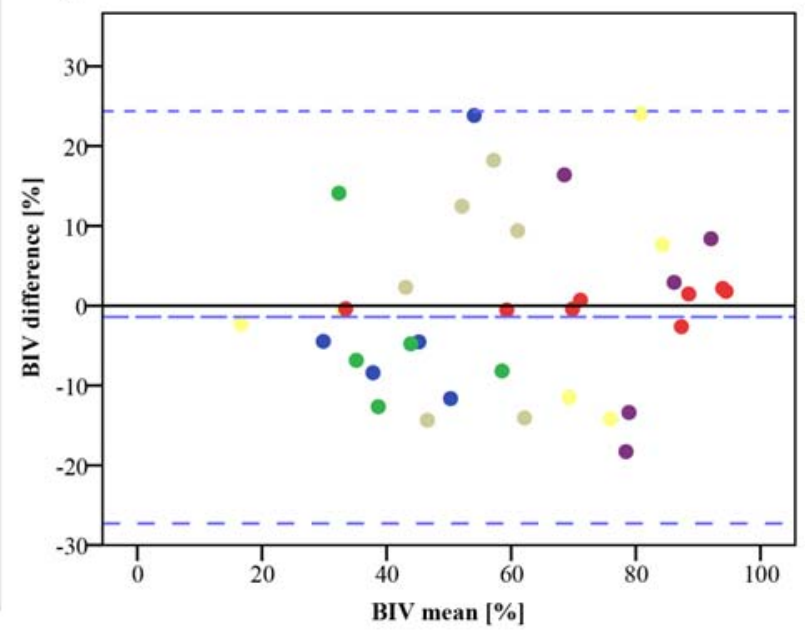

Fig. 5. (a) Relationship of the bone-implant contact (BIC) measurement between the histological data and the SR $\mu \mathrm{CT}$ data with the line of equality. Data of the same samples are shown in equal colours. (b) Bland Altman plot of the difference between the histological and SR $\mu$ CT BIC measurements against their mean value. Mean difference (long dash) describing the bias between the methods. Short dashed lines indicate limits of agreement. (c) Relationship of the bone-implant volume (BIV) measurement between the histological data and the SR $\mu$ CT data with the line of equality. (d) Bland Altman plot of the difference between the histological and SR $\mu$ CT BIV measurements against their mean value.

bone can be clearly separated on the image using a fitting threshold from the absorption histograms.

If the implant surface is predominantly covered by bone tissue the partial volume effect in SR $\mu \mathrm{CT}$ measurements influences the threshold BIC values and may also lead to a mixture of bone/implant absorption values above the constantly defined $18 \mu \mathrm{m}$ distance of analysis. These absorption values, which are higher compared to mineralised bone, were filtered by the threshold procedure.

Due to the partial volume effect the BIC line of measurement for $\mathrm{SR} \mu \mathrm{CT}$ can be 1 - 2 pixels away from the histological line of measurement. This can influence the $\mathrm{SR} \mu \mathrm{CT}$ BIC value, as separated thin regions of mineralised tissue, which are part of the histological BIC value, would not be counted. Associated to this effect, Fig. 5a shows values where the histological BIC is about $100 \%$ and where the BIC of the SR $\mu \mathrm{CT}$ is noticeably lower. However, these conditions were not prevalent in our study and do not result in significant differences of the BIC between SR $\mu \mathrm{CT}$ and histomorphometry.

If a small soft tissue layer on the implant is followed by mineralised bone, this would not be measured as BIC in histology and would cause an overestimation of BIC in $\mathrm{SR} \mu \mathrm{CT}$. In this study, there were no such cases found within the BIC analysis (Fig. 5a). Figs. 4g-h gives an example of the direct contact of bone tissue with the implant surface. In order to compare the BIC between $\mathrm{SR} \mu \mathrm{CT}$ slices and histological sections it has to be ensured that the ROI selection (Fig. 1b) is as identical to histology as possible.

Other studies (Park et al., 2005; Rebaudi et al., 2004) have also compared the histomorphometric BIC of dental implants to 3D measurements, using a conventional $\mu \mathrm{CT}$. Rebaudi et al. (2004) showed a maximum difference of $16 \%$ mean BIC. In contrast to our study, a $45 \mu \mathrm{m}$ thick peri-implant boundary served for the BIC measurement. Because of the monochromatic character of the X-rays 
a
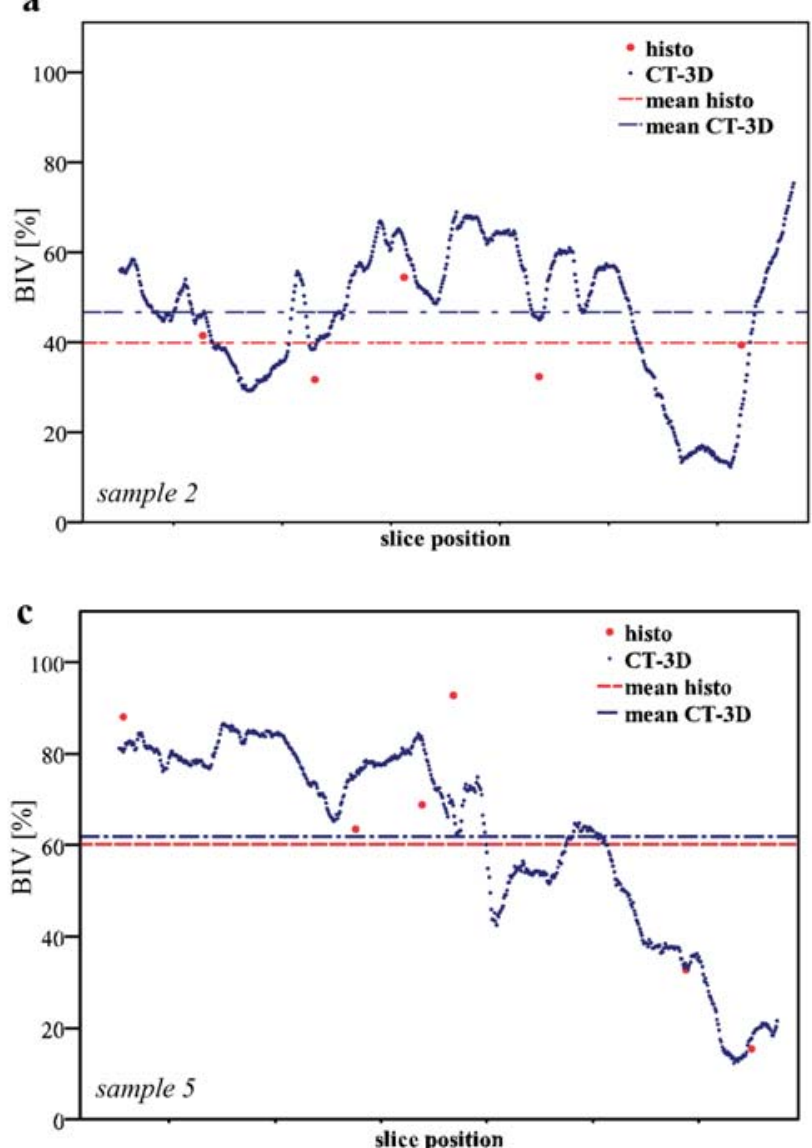

b

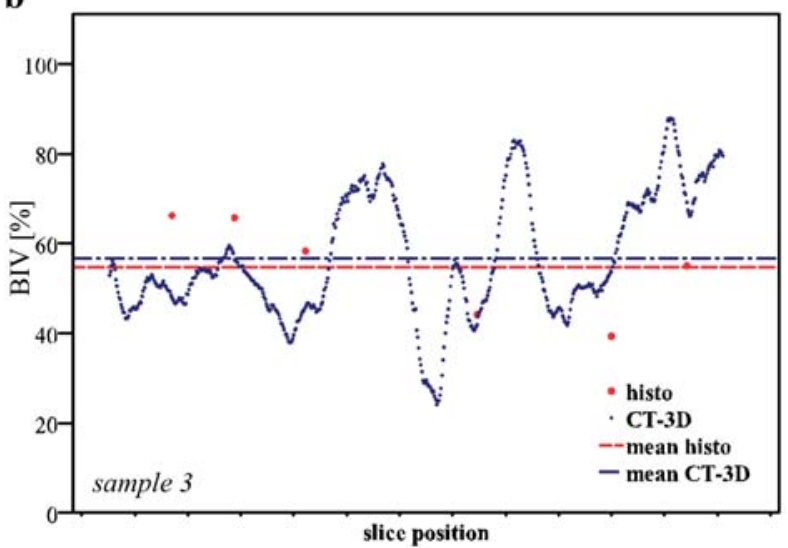

d

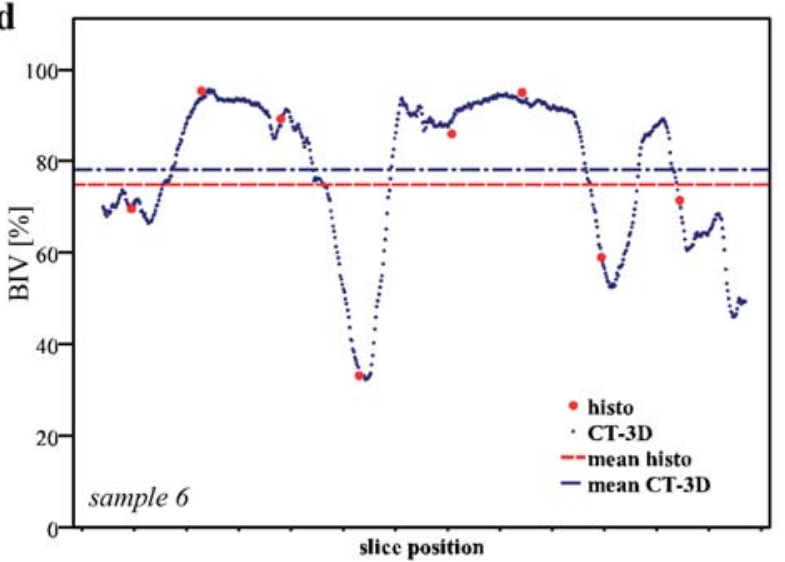

Fig. 6. BIV analysis for selected samples along the histological orientation shows a good agreement between the single values from histomorphometry (red dots) and the continuous data from the SR $\mu \mathrm{CT}$ analysis (blue dots). Although there is a high variability of the intra-sample BIV, the mean values of the BIV are nearly the same for 3-4 histological sections against the whole bone volume in the cavity analysed with SR $\mu \mathrm{CT}$ (b-d). With a bone volume of about $50 \%$, the overlap of similar absorption values between embedding material and low mineralised bone becomes significant and leads to a higher amount of BIV detected by SR $\mu \mathrm{CT}$ analysis (a).

and the high photon density, our SR $\mu \mathrm{CT}$ measurements enabled the use of a distance of $18 \mu \mathrm{m}$, which more closely represents the implant surface in contact with bone (Bernhardt et al., 2004; Bernhardt et al., 2005). It was found that of the type of bone has an influence from where the BIC originates (Ivanoff et al., 1997). Our study focused on newly formed spongious bone structures.

Another study performed BIC measurements with conventional $\mu \mathrm{CT}$ and histomorphometry, without however selecting identical slice positions (Park et al., 2005). They showed a correlation in BIC between the two methods with an underestimation of $10 \%$ for $\mu \mathrm{CT}$ data in relation to histomorphometry. These two studies suffered from the influence of the partial volume effect around the trabecular boundaries and beam hardening near the implant surface using conventional $\mu \mathrm{CT}$. These problems of conventional $\mu \mathrm{CT}$ measurements of peri-implant bone response in correlation to histomorphometry were also shown by Jansen et al. (2009).

With the knowledge gained from this analysis of BIC, we made two main conclusions. First, the application of high resolution $\mathrm{SR} \mu \mathrm{CT}$ has the potential to detect BIC in close proximity to the implant surface, with the overall results being comparable to histomorphometric findings.
Secondly, using the full SR $\mu$ CT 3D-data with about 360 CT positions per implant, the comparison with four histological sections showed a non-significant difference of only 4.9 $\%$. On the basis of our results using a maximum of eight areas of measurement per implant (two areas/section, four sections/implant) we concluded that histomorphometry is an appropriate method to demonstrate pronounced differences of BIC. As mentioned earlier, in many cases only one or two histological sections per implant can be used for histomorphometric evaluation. This can lead to an over- or underestimation of the BIC. In case expected significant differences are lower than $5 \%$, SR $\mu \mathrm{CT}$ could give additional information on the performance of different surfaces.

The analysis of the BIV gives information about the thickness of newly formed peri-implant bone, which is of interest in correlation to the determined BIC. Differences in bone volume are of interest when comparing different implant surfaces and their possible influence on bone formation, e.g. by the application of surface-integrated growth factors.

In a previous study we showed a reasonable agreement of peri-implant bone morphology between SR $\mu \mathrm{CT}$ and histomorphometry (Bernhardt et al., 2004). Because of 
the loss of information during the histological sawing and grinding process, we concluded that differences in bone formation could become questionable. This is also described by other studies (Sennerby et al., 2001). In contrast to these earlier results, we could show in this study that information from 3-4 histological sections per implant ( 2 areas/section) leads to reasonable results in BIV analysis. In another study (Chappard et al., 2005), histomorphometry of 70 bone biopsies of patients suffering from metabolic bone diseases were compared to conventional 3D- $\mu \mathrm{CT}$.

Stiller et al. (2009) compared the validity of histomorphometry to CT-2D and CT-3D (SR $\mu \mathrm{CT}$ ), analysing bone biopsies without implants. Bone area fractions determined by histomorphometry and the corresponding slice from CT-2D also showed a good agreement, with a minor difference of only $1.6 \%$. Comparing histomorphometry to CT-3D, a maximum difference of $8.5 \%$ was shown. These results are very similar to our findings.

The SR $\mu$ CT-measurement was done after PMMA embedding. This was a prerequisite to preserve the morphology of the tissues over time. The absorption histograms for PMMA values were found to be in the same range as soft tissue and low mineralised bone. A high amount of low mineralised bone thus can lead to an underestimation of detected bone tissue, as there will be insufficient contrast between PMMA and low mineralised bone tissue.

The maximum intra-sample difference of BIV in our study showed a non-significant difference between histomorphometry and SR $\mu \mathrm{CT}$. The difference between histomorphometry compared to CT-2D and compared to CT-3D was only $1.2 \%$. Based on these results, we conclude that the BIV values gained from 3-4 histological sections per implant/bone sample represent solid data with only minor differences to the 3D situation (Fig. 6a-d).

An advantage of $\mathrm{SR} \mu \mathrm{CT}$ in contrast to conventional $\mu \mathrm{CT}$ is the reduction of the partial volume effect for trabecular boundaries, thus facilitating threshold setting. As the PVE is related to the CT image resolution, the effect could be reduced with high-resolution $\mu \mathrm{CT}$-scanners and sufficient X-ray intensity. For pure bone biopsies, conventional $\mu \mathrm{CT}$ should be able to replace conventional histomorphometry in the measurement of bone volume and microstructure of trabecular bone (Uchiyama et al., 1997). The analysis of a highly absorbent osseointegrated implant, when measuring the BIC, is more feasible using a SR $\mu \mathrm{CT}$ (Bernhardt et al., 2004).

This study demonstrated that 3-4 histological sections allow the demonstration of differences in BIC and BIV, without major disagreement with synchrotron measurements. Although histomorphometry is a timeconsuming procedure, it has the advantage of revealing information from non-mineralised bone tissue. Such tissue is invisible to $\mu \mathrm{CT}$ when scanning highly absorbent materials like osseointegrated dental implants. A limiting factor of $\mathrm{SR} \mu \mathrm{CT}$ is the availability of synchrotron radiation facilities for such experiments.

The question arises of what is meant by a clinically important difference in BIC. It would be preferable for clinically significant differences in BIC to be supported by significant differences in other areas, e.g. mechanics. Various studies have tried to correlate biomechanical data to histomorphometric data (Buser et al., 2004; Ferguson et al., 2006). A major disadvantage of biomechanical testing is the destructive nature of methods, like removal torque testing. In such cases $\mathrm{SR} \mu \mathrm{CT}$ with its non-destructive character would enable a consecutive test method of the same implant/bone sample. Because of the digital 3D representation of peri-implant bone in $\mathrm{SR} \mu \mathrm{CT}$ it is possible to simulate mechanical behaviour of the implant with the help of finite element models. Further data, e.g. the degree of mineralisation within newly formed bone, can be three dimensionally determined (Campbell et al., 2011).

\section{Acknowledgements}

This study has been funded by the German Research Foundation DFG (Deutsche Forschungsgemeinschaft) under the Transregio SFB 67 and the BMBF project (05 ES3XBA/5). Further we thank Dr. Jürgen Goebbels for his assistance in synchrotron experiments, Mrs. Andrea Groß for the graphical layout and Dr. Ian Chambers for the English editing.

\section{References}

Albrektsson T, Branemark PI, Hansson HA, Lindstrom J (1981) Osseointegrated titanium implants. Requirements for ensuring a long-lasting, direct bone-to-implant anchorage in man. Acta Orthop Scand 52: 155-170.

Albrektsson T, Jacobsson M (1987) Bone-metal interface in osseointegration. J Prosthet Dent 57: 597-607.

Bernhardt R, Scharnweber D, Muller B, Thurner P, Schliephake H, Wyss P, Beckmann F, Goebbels J, Worch H (2004) Comparison of microfocus- and synchrotron X-ray tomography for the analysis of osteointegration around Ti6Al4V implants. Eur Cell Mater 7: 42-51.

Bernhardt R, van den Dolder J, Bierbaum S, Beutner R, Scharnweber D, Jansen J, Beckmann F, Worch H (2005) Osteoconductive modifications of Ti-implants in a goat defect model: characterization of bone growth with SRÊCT and histology. Biomaterials 26: 3009-3019.

Branemark PI (1983) Osseointegration and its experimental background. J Prosthet.Dent 50: 399-410.

Buser D, Broggini N, Wieland M, Schenk RK, Denzer AJ, Cochran DL, Hoffmann B, Lussi A, Steinemann SG (2004) Enhanced bone apposition to a chemically modified SLA titanium surface. J Dent.Res 83: 529-533.

Campbell GM, Bernhardt R, Scharnweber D, Boyd SK (2011) The bone architecture is enhanced with combined PTH and alendronate treatment compared to monotherapy while maintaining the state of surface mineralization in the OVX rat. Bone 49: 225-232.

Chappard D, Retailleau-Gaborit N, Legrand E, Basle MF, Audran M (2005) Comparison insight bone measurements by histomorphometry and microCT. J Bone Miner Res 20: 1177-1184.

Donath K, Breuner G (1982) A method for the study of undecalcified bones and teeth with attached soft tissues. 
The Sage-Schliff (sawing and grinding) technique. J Oral Pathol 11: 318-326.

Ferguson SJ, Broggini N, Wieland M, de Wild M, Rupp F, Geis-Gerstorfer J, Cochran DL, Buser D (2006) Biomechanical evaluation of the interfacial strength of a chemically modified sandblasted and acid-etched titanium surface. J Biomed Mater Res A 78: 291-297.

Ivanoff CJ, Sennerby L, Johansson C, Rangert B, Lekholm U (1997) Influence of implant diameters on the integration of screw implants. An experimental study in rabbits. Int J Oral Maxillofac Surg 26: 141-148.

Jansen JA, Schouten C, Meijer GJ, van den Beucken JJJP, Spauwen PHM (2009) The quantitative assessment of peri-implant bone responses using histomorphometry and micro-computed tomography. Biomaterials 30: 4539-4549.

Johansson CB, Hansson HA, Albrektsson T (1990) Qualitative interfacial study between bone and tantalum, niobium or commercially pure titanium. Biomaterials 11: 277-280.

Linder L, Albrektsson T, Branemark PI, Hansson HA, Ivarsson B, Jonsson U, Lundstrom I (1983) Electron microscopic analysis of the bone-titanium interface. Acta Orthop Scand 54: 45-52.

Liu S, Broucek J, Virdi AS, Sumner DR (2012) Limitations of using micro-computed tomography to predict bone-implant contact and mechanical fixation. J Microsc 245: 34-42.

Oyonarte R, Pilliar RM, Deporter D, Woodside DG (2005) Peri-implant bone response to orthodontic loading: Part 1. A histomorphometric study of the effects of implant surface design. Am J Orthod Dentofacial Orthop 128: 173 181.

Park YS, Yi KY, Lee IS, Jung YC (2005) Correlation between microtomography and histomorphometry for assessment of implant osseointegration. Clin Oral Implants Res 16: 156-160.

Rebaudi A, Koller B, Laib A, Trisi P (2004) Microcomputed tomographic analysis of the peri-implant bone. Int J Periodontics Restorative Dent 24: 316-325.

Sennerby L, Wennerberg A, Pasop F (2001) A new microtomographic technique for non-invasive evaluation of the bone structure around implants. Clin Oral Implants Res 12: 91-94.

Stadlinger B, Hintze V, Bierbaum S, Moller S, Schulz MC, Mai R, Kuhlisch E, Heinemann S, Scharnweber D, Schnabelrauch M, Eckelt U (2011) Biological functionalization of dental implants with collagen and glycosaminoglycans. A comparative study. J Biomed Mater Res B Appl Biomater 100B: 331-341.

Stiller M, Rack A, Zabler S, Goebbels J, Dalugge O, Jonscher S, Knabe C (2009) Quantification of bone tissue regeneration employing beta-tricalcium phosphate by threedimensional non-invasive synchrotron micro-tomography - a comparative examination with histomorphometry. Bone 44: 619-628.

Uchiyama T, Tanizawa T, Muramatsu H, Endo N, Takahashi HE, Hara T (1997) A morphometric comparison of trabecular structure of human ilium between microcomputed tomography and conventional histomorphometry. Calcif Tissue Int 61: 493-498.

\section{Discussion with Reviewers}

Reviewer I: What is the final advantage of $\mu \mathrm{CT}$ analysis compared with histology?

Authors: Providing sufficient availability, the advantage of $\mu \mathrm{CT}$ analysis compared to histology is a fast and nondestructive analysis of samples with three-dimensional data and high statistical power. Furthermore, there is no tissue preparation necessary for the measurement of mineralised bone samples. The special character of the $\mu \mathrm{CT}$ information provides the possibility to apply more than one method. Next to histology, other analytical methods like FEM investigation or spatial pore analysis are feasible. The challenge for desktop $\mu \mathrm{CT}$ systems still is to reduce metal induced artifacts.

Reviewer I: Is it not better to examine the specimens fresh (without fixation) using $\mu \mathrm{CT}$ in order to avoid artifacts due to fixation and embedding?

Authors: It is true that a measurement of fresh samples would be favourable. In most cases this can be done using desktop $\mu \mathrm{CT}$ systems due to their availability. For SR $\mu \mathrm{CT}$, which has been used in this study, it was hard to plan the synchrotron beam-time between animal experiment and histological preparation. The reason for the fixation of tissues as applied in this study is the necessity of an unchanged morphological agreement of the bone/implant interface between both measurements ( $\mathrm{SR} \mu \mathrm{CT} / \mathrm{histology})$ for the time of investigation.

Reviewer III: What are the correlation coefficients of the linear regressions between histology and SR $\mu$ CT-based measurements of BIC and BIV? What are the main sources for discrepancies between the two methods?

Authors: The correlation coefficients between SR $\mu$ CT and histology are $\mathrm{R}=0.97$ for $\mathrm{BIC}$ and $\mathrm{R}=0.83$ for $\mathrm{BIV}$. The main aspect for BIC differences is the partial volume effect in the $\mathrm{SR} \mu \mathrm{CT}$ at the bone/implant interface. This shifts the distance between histological and SR $\mu \mathrm{CT}$ line of measurement toward the peri-implant tissues. If the implant is mostly covered with a bone layer being thinner than the PVE (in our study $<18 \mu \mathrm{m}$ ), this will result in an underestimation of bone in $\mathrm{SR} \mu \mathrm{CT}$ analysis. In our study this effect did not lead to a significant change within the $\mathrm{BIC}$ between histology and SR $\mu \mathrm{CT}$. An important factor for discrepancies of BIC between histomorphometry and $\mathrm{SR} \mu \mathrm{CT}$ may also be the matching of the ROI positions in the images being compared. For BIV measurement, PMMA fixation of the samples, which was essential for the preservation of tissue morphology, complicates the finding of an appropriate threshold, only being associated with newly formed bone. This can also affect the BIC and BIV values.

Reviewer III: In addition to BIC and BIV, would it be possible to measure the degree of mineralisation of bone around the implant with SR $\mu \mathrm{CT}$ ? Or would the presence of the implant hamper this quantification?

Authors: The measurement of the degree of mineralisation by $\mathrm{SR} \mu \mathrm{CT}$ using bone absorption histograms is possible. 
In the magnified SR $\mu \mathrm{CT}$ image Fig. 4h, different grey (absorption) values of bone are visible. These can be used for the classification of the mineral density close to the implant surface, as there were no metal induced artifacts detectable.

Reviewer III: If BIC and BIV are assessed with desktop micro-computed tomography at the same resolution reported in the present study, would it be possible to estimate the "exclusion zone" where image artefacts would not allow a proper analysis?
Authors: A proper distance from the implant surface for the analysis of BIC using a desktop $\mu \mathrm{CT}$ system depends on the extension of metal induced artifacts around the implant. The artifacts are related to the applied hardware (e.g. implant material, shape and thickness, X-ray filter and X-ray intensity, and the software (e.g. reconstruction algorithm and beam hardening filtering). Using a $\mathrm{CaP} /$ implant phantom of appropriate size, the 'exclusion zone' of applied $\mu \mathrm{CT}$ systems can be estimated by a line scan analysis through the bone/implant interface. 